\title{
Brain Derived Neurotrophic Factor Levels in Aged Rats Post-Systemic Human Mesenchymal Stem Cell Administration
}

\section{Adisti Dwijayanti, ${ }^{1,2}$, Wildan Mubarok ${ }^{3,4}$, Arief Boediono ${ }^{5}$, Jan Sudir Purba ${ }^{6}$, Erni Hernawati Purwaningsih ${ }^{2}$, Fransiscus Dhyanagiri Suyatna ${ }^{7}$}

\author{
${ }^{1}$ Doctoral Program in Biomedical Sciences, Faculty of Medicine, Universitas Indonesia \\ ${ }^{2}$ Department of Medical Pharmacy, Faculty of Medicine, Universitas Indonesia \\ ${ }^{3}$ Department of Biology, Faculty of Mathematics and Natural Sciences, Bogor Agricultural \\ University \\ ${ }^{4}$ Stem Cells \& Tissue Engineering Research Center, Indonesian Medical Education and \\ Research Institute, Faculty of Medicine, Universitas Indonesia \\ ${ }^{5}$ Department of Anatomy, Physiology, and Pharmacology, Faculty of Veterinary Medicine, \\ Bogor Agricultural University \\ ${ }^{6}$ Department of Neurology, Faculty of Medicine, Universitas Indonesia \\ ${ }^{7}$ Department of Clinical Pharmacology and Therapeutics, Faculty of Medicine, Universitas \\ Indonesia \\ *Corresponding author: adisti.dwijayanti@ui.ac.id
}

\begin{abstract}
Brain-Derived Neurotrophic Factor (BDNF) levels were affected by aging. Brain BDNF levels were known to decrease along with advanced age thus correlated with any diseases such as cognitive impairment and Alzheimer. Mesenchymal Stem Cell (MSC) is one of the potential modalities actively investigated against age-related diseases. This study evaluated the effect of human MSC administration to brain BDNF levels in aged rats. Intravenous injection of 10 million per body weight human MSC were given four times in 3 months interval to 22-24 months old female and male Spraque-Dawley rats. As control group, aged rats were injected by normal saline at the same volume and frequencies. Moreover, young 3-6 months rats also examined as negative control. By the end of the experiment, we analyzed three rats from each group. Brain BDNF levels were measured by enzyme-linked immunosorbent assay and normalize to the protein levels. One-way ANOVA and LSD post hoc analysis was performed to compare the differences between groups. BDNF levels in male appeared similar between young, aged, and MSC treated groups. Meanwhile, control aged female groups had significantly lower BDNF levels compared to young $(\mathrm{p}=0.019)$ and MSC-treated aged rats $(\mathrm{p}=0.001)$. There was no difference of BDNF levels between young and MSC-treated aged in female rats $(p=0,068)$. Both sex had similar BDNF levels ( $p=0.249)$ in control-aged groups. In contrast, female young and MSC-treated aged rats achieved significantly higher BDNF levels $(\mathrm{p}=0.009$ and $\mathrm{p}<0.001$ ) compared to the male groups, respectively. These results suggest that human mesenchymal stem cell intravenous injection can increase brain BDNF levels in female aged rats.
\end{abstract}

Keywords: BDNF, human mesenchymal stem cell, aged rats. 


\section{INTRODUCTION}

One of the major molecules in ageing is Brain-Derived Neurotrophic Factor (BDNF). Study in human suggested that plasma BDNF is a biomarker of impaired memory and general cognitive function in ageing women (Komulainen et al, 2008). BDNF is highly expressed in brain areas demonstrating functional plasticity (Tapia-Arancibia et al, 2008). Brain BDNF levels were known to decrease along with advanced age thus correlated with any diseases such as cognitive impairment and Alzheimer (Budni et al, 2015). Cognitive defects associated with aging might be related to BDNF system (Calabrese, 2013). Restoring BDNF levels may be promising therapeutic target in preventing age-related diseases. Mesenchymal Stem Cell (MSC) role in aging was being enormously investigated against age-related diseases. Human adipose tissuederived MSC (ADMSCs) intravenous injection in aged male ICR mice (18-month-old) able to

\section{METHODS}

The protocol of this study has been approved by the Animal Care and Use Committee Veterinary Hospital Faculty of Veterinary Bogor Agricultural Institute (N0: 212016 ACUC RSHP FKH-IPB). Female and male, 22-24 months old Spraque-Dawley rats were housed individually, fed ad libitum in 12hour light/dark cycle. WJ-MSC used in this study was prepared according to De Bruyn et al (2011).

The aged rats were divided into 2 groups: positive control group and WJ-MSC treatment group. The WJ-MSC treatment groups restore whole brain BDNF levels and showed cognitive improvement (Park et al, 2013). Human amniotic membrane-derived MSC and ADMSCs injection in 10-month-old male F344 rats elongate health span and life span along with neurotrophic factors recovery (Kim et al, 2015). Another source of MSC, Wharton-Jelly (WJ) MSC, which is easier to obtain may also has promising potential advantage for treatment of age-related diseases.

In human studies, plasma BDNF associated with general cognitive decline in ageing women but not in men (Komulainen et al, 2008). Moreover, Chan and Ye (2017) considered sex influences in BDNF levels on sex hormones, which were able to modulate the activities of BDNF. Thus, evaluating BDNF in both sexes might be valuable. This study evaluated the effect of human WJ-MSC administration to brain BDNF levels in female and male aged rats.

were further divided into 4 different doses: $10^{6}$ per $\mathrm{kg}$ body weight four times at 3-month intervals, $10^{7}$ per kg body weight four times at 3 month intervals, $10^{6}$ per kg body weight twice at 6-month intervals, and $10^{7}$ per $\mathrm{kg}$ body weight twice at 6-month intervals. Normal saline were injected to the positive control group in the same volume and intervals as WJ-MSC treatment groups.

After one year, the aged rats were sacrificed. Blood and organs were collected and stored for further analysis. Brain BDNF levels were measured by enzyme-linked immunosorbent assay (Elabscience Catalog No: 
E-EL-R1235) and normalize to the brain protein levels. BDNF levels are expressed in $\mathrm{pg} / \mathrm{mg}$ protein. The results were compared to young rats 3-6 months old. One-way ANOVA and LSD

\section{RESULTS}

At the end of our experiment, the mortality rate of the WJ-MSC treatment groups was high. Thus in this report, we analyzed one of post hoc analysis was performed to compare the differences between groups. Statistic analyses were prepared using SPSS 20.

the WJ-MSC treatment groups dose $10^{7}$ per $\mathrm{kg}$ body weight four times at 3-month intervals. We evaluated three rats from each group.
A.

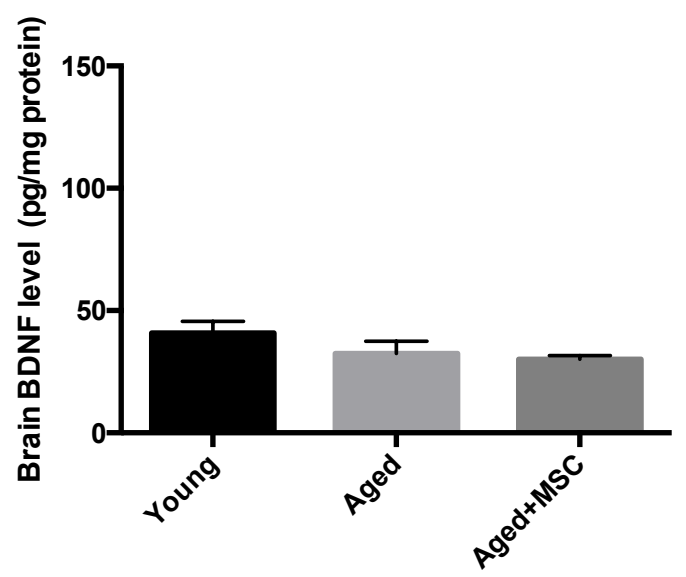

B.

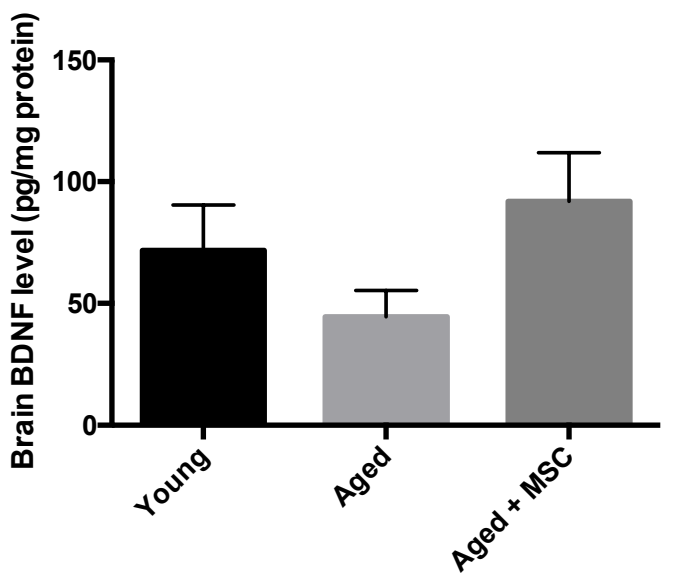

Figure 1. BDNF levels in A: male rats; B: female rats $(n=3)$.

Values are presented in mean \pm SD.

BDNF levels in male appeared similar between young, aged, and MSC treated groups (Figure 1). Meanwhile, control aged female groups had significantly lower BDNF levels compared to young $(\mathrm{p}=0.019)$ and MSC-treated aged rats $(p=0.001)$. There was no difference of BDNF levels between young and MSC-treated aged in female rats $(p=0,068)$. Both sex had similar BDNF levels $(\mathrm{p}=0.249)$ in control-aged groups. In contrast, female young and MSCtreated aged rats achieved significantly higher BDNF levels ( $p=0.009$ and $p<0.001)$ compared to the male groups, respectively.

\section{DISCUSSION}

Our research showed the differences in male and female aged rats after repetitive $\mathrm{WJ}$ MSC intravenous injection. Brain BDNF levels in female aged rats markedly increased after human WJ-MSC intravenous injection but not in the male rats. This sex difference response might be related to the role of sex hormones as previously studied (Chan and Ye, 2017). Estrogen receptors are found in the brain (hippocampus and frontal lobes), these two areas are important for cognitive function. Research 
about the estrogen role in BDNF expression is still in controversial.

Other factors could also influences BDNF expression in the brain. Senescence causes loss of endogenous rhythms of BDNF expression. This is associated with structural, molecular, and cellular changes of the aged brain thus lead to alterations in circadian clock (CoriaLucero 2016). The location of the brain sample measured for BDNF also may influence the

\section{CONCLUSION}

In conclusion, WJ-MSC intravenous injection increased brain BDNF levels in female aged rats.

\section{REFERENCES}

Bettio LEB, Rajendran L, Gil-Mohapel J. 2017. The effects of aging in the hippocampus and cognitive decline. J Neu Bio Rev. 79:66-86.

Budni J, Bellettini-Santos T, Mina F, Garcez ML, Zugno AI. 2015. The involvement of BDNF, NGF and GDNF in aging and alzheimer's disease. Aging Dis. 6(5): $331-41$.

Chan CB and Ye K. 2017. Sex differences in brain-derived neurotrophic factor signaling and functions. J Neurosci Res. 95(1-2): 328-35.

Coria-lucero CD, et al. 2016. Rhythmic bdnf and trkb expression patterns in the prefrontal cortex are lost in aged rats. Brain Res. 1653: 51-8.

De Bruyn C, et al. 2011. A rapid, simple, and reproducible method for the isolation of
BDNF levels. Along with the cerebral cortex, brain BDNF Deficits also mainly found in hippocampus region of the aged mice (Betho et al 2017). Our study examined the BDNF levels from the frontal cerebral cortex, area that also has important role in cognitive function. Moreover, there also a link between BDNF expression and housing of the rats as explained by Murinova et al (2017).

mesenchymal stromal cells from Wharton's jelly without enzymatic treatment. Stem Cells Dev. 20(3): 54757.

Kim D, et al. 2015. Health span-extending activity of human amniotic membraneand adipose tissue-derived stem cells in F344 rats. Stem Cells Transl Med. 4(10): 1144-54.

Komulainen P, et al. 2008. BDNF is a novel marker of cognitive function in ageing women: the DR's EXTRA study. Neurobiol Learn Mem. 90: 596-603.

Murinova J, Hlavacova N, Chmelova M, Riecansky I. 2017. The evidence for altered BDNF expression in the brain of rats reared or housed in social isolation: a systematic review. Front Behav Neurosci. 11: 101.

Park D, et al. 2013. Human adipose tissuederived mesenchymal stem cells improve cognitive function and physical activity in ageing mice. J Neurosci Res. 91: 660-70. 